\title{
FREE CUMULANTS OF SOME PROBABILITY MEASURES
}

\author{
MELANIE HINZ \\ Institut für Mathematik und Informatik \\ Ernst-Moritz-Arndt Universität Greifswald \\ Jahnstrasse 15a, D-17487 Greifswald, Germany \\ E-mail: melanie.hinz@uni-greifswald.de \\ WOJCIECH MŁOTKOWSKI \\ Institute of Mathematics, Wrocław University \\ Pl. Grunwaldzki 2/4, 50-384 Wroctaw, Poland \\ E-mail:mlotkow@math.uni.wroc.pl
}

1. Preliminaries. Let $X$ be a finite, linearly ordered set. By a noncrossing partition of $X$ we will mean a collection $\pi$ of nonempty, pairwise disjoint subsets (called blocks of $\pi)$ such that $\bigcup \pi=X$, which satisfies the following condition: if $x_{1}<x_{2}<x_{3}<x_{4}$, with $x_{1}, x_{3} \in U_{1} \in \pi$ and $x_{2}, x_{4} \in U_{2} \in \pi$, then $U_{1}=U_{2}$. The class of all noncrossing partitions of $X$ will be denoted by $\mathrm{NC}(X)$. We also define $\mathrm{NC}_{1,2}(X)$ as the family of those $\sigma \in \mathrm{NC}(X)$ for which every block has at most 2 elements. We will write $\mathrm{NC}(m)$ and $\mathrm{NC}_{1,2}(m)$ instead of $\mathrm{NC}(\{1,2, \ldots, m\})$ and $\mathrm{NC}_{1,2}(\{1,2, \ldots, m\})$.

Every $\pi \in \mathrm{NC}(X)$ admits a natural partial order. Namely, for $U, V \in \pi$ we write $U \prec V$ whenever there are $r, s \in V$ such that $r<k<s$ holds for every $k \in U$. We define the depth of a block as $d(U, \pi):=|\{V \in \pi: U \prec V\}|$. A block is called outer if $d(U, \pi)=0$, otherwise it is called inner. Note that for every inner block $U \in \pi$ there is a unique block in $\pi$, denoted by $U^{\prime}$, such that $U \prec U^{\prime}$ and $d(U, \pi)=d\left(U^{\prime}, \pi\right)+1$. We also define derivatives of higher orders by $V^{(0)}:=V$ and $V^{(k)}:=\left(V^{(k-1)}\right)^{\prime}$.

2000 Mathematics Subject Classification: Primary 46L54; Secondary 60 C05.

Key words and phrases: noncrossing partitions, free cumulants, Jacobi coefficients.

Research of W. Młotkowski has been supported by KBN grant 1P03A 01330, by a Marie Curie Transfer of Knowledge Fellowship of the European Community's Sixth Framework Programme under contract number MTKD-CT-2004-013389 and by 7010 POLONIUM project "NonCommutative Harmonic Analysis with Applications to Operator Spaces, Operator Algebras and Probability".

The paper is in final form and no version of it will be published elsewhere. 
Let $\mu$ be a compactly supported probability measure on the real line, with the moment sequence

$$
s_{m}(\mu):=\int_{t \in \mathbb{R}} t^{m} d \mu(t) .
$$

Then there is a unique sequence $\left\{P_{m}(x)\right\}_{m=0}^{\infty}$ of monic polynomials, with $\operatorname{deg} P_{m}=m$, which are orthogonal with respect to $\mu$. It is known that they satisfy the recurrence relation: $P_{0}(x)=1$ and for $m \geq 0$

$$
x P_{m}(x)=P_{m+1}(x)+\beta_{m} P_{m}(x)+\gamma_{m-1} P_{m-1}(x),
$$

under convention that $P_{-1}=0$, where the Jacobi coefficients satisfy: $\beta_{m} \in \mathbb{R}, \gamma_{m} \geq 0$ and if $\gamma_{m}=0$ for some $m$ then $\gamma_{n}=\beta_{n}=0$ for every $n>m$ (see [Ch]). These coefficients show up in the continued fraction expansion of the Cauchy transform of $\mu$, namely:

$$
G_{\mu}(z):=\int_{t \in \mathbb{R}} \frac{d \mu(t)}{t-z}=\frac{1}{z-\beta_{0}-\frac{\gamma_{0}}{z-\beta_{1}-\frac{\gamma_{1}}{z-\beta_{2}-\frac{\gamma_{2}}{z-\beta_{3}-\frac{\gamma_{3}}{\ddots}}}} .}
$$

There is a combinatorial formula, due to Accardi and Bożejko, connecting moments and the Jacobi coefficients of $\mu$, namely

$$
s_{m}(\mu)=\sum_{\sigma \in \mathrm{NC}_{1,2}(m)} \prod_{\substack{V \in \sigma \\|V|=1}} \beta_{d(V, \sigma)} \prod_{\substack{V \in \sigma \\|V|=2}} \gamma_{d(V, \sigma)} .
$$

Another important numbers related to $\mu$ are the free cumulants $r_{m}(\mu), m \geq 1$ (see $[\mathrm{S} 1, \mathrm{~S} 2])$, which are defined by:

$$
s_{m}(\mu)=\sum_{\pi \in \mathrm{NC}(m)} \prod_{U \in \pi} r_{|U|}(\mu) .
$$

Their generating function

$$
R_{\mu}(z):=\sum_{k=0}^{\infty} r_{k+1}(\mu) z^{k}
$$

is called the $R$-transform. These two functions are related by

$$
\frac{1}{G_{\mu}(z)}=z-R_{\mu}\left(G_{\mu}(z)\right) \text {. }
$$

From now on we will confine ourselves to a special class of measures $\mu$. For $a>0$, $b \geq 0$ and $u, v \in \mathbb{R}$ we define $\mu(a, b, u, v)$ as the unique $\mu \in \mathcal{M}$ such that its Jacobi coefficients are:

$$
\gamma_{m}=\left\{\begin{array}{l}
a \text { if } m=0 \\
b \text { if } m \geq 1
\end{array}\right.
$$




$$
\beta_{m}=\left\{\begin{array}{l}
u \text { if } m=0, \\
v \text { if } m=1, \\
v \text { if } m>1 \text { and } b>0 \\
0 \text { if } m>1 \text { and } b=0 .
\end{array}\right.
$$

This class of measures was first studied by Cohen and Trenholme [CT] from the point of view of harmonic analysis. Then Saitoh and Yoshida studied them from the point of view of free probability. For $\mu=\mu(a, b, u, v)$ they calculated the Cauchy transform:

$$
G_{\mu}(z)=\frac{2 b(z-u)-a(z-v)-a \sqrt{(z-v)^{2}-4 b}}{2 b(z-u)^{2}-2 a(z-u)(z-v)+2 a^{2}}
$$

and the $R$-transform:

$$
R_{\mu}(w)=u+\frac{a w}{1+(u-v) w}
$$

if $a=b$ and

$$
R_{\mu}(w)=u+\frac{a}{b-a} \frac{1-(v-u) w+\sqrt{((v-u) w-1)^{2}-4(b-a) w^{2}}}{2 w}
$$

otherwise. For $a \leq b$ the authors of [SY] found the Lévy-Khinchin formula:

$$
R_{\mu}(z)=u+a \int_{\mathbb{R}} \frac{z}{1-t z} d \nu(t)
$$

where if $a=b$ then $\nu=\delta_{v-u}$ and if $a<b$ then $\nu$ is absolutely continuous with density

$$
\frac{1}{2 \pi(b-a)} \sqrt{4(b-a)-(t-(v-u))^{2}}
$$

on the interval $(t-(v-u))^{2} \leq 4(b-a)$. Basing on this they proved that $\mu(a, b, u, v)$ is infinitely divisible with respect to the free convolution if and only if $a \leq b$. Bożejko and Bryc [BB] observed that one can use (13) to find the free cumulants of $\mu(a, b, u, v)$ when $a \leq b$. Now, since every free cumulant $r_{m}(\mu(a, b, u, v))$ is a polynomial in $a, b, u, v$, the resulting formula holds for all $\mu(a, b, u, v)$. The aim of this paper is to find the free cumulants of $\mu(a, b, u, v)$ in a purely combinatorial way.

2. The result. Now we are ready to state the result.

THEOREM. For the free cumulants $r_{m}:=r_{m}(\mu(a, b, u, v))$ we have: $r_{1}=u$ and for $n \geq 1$

$$
\begin{aligned}
r_{2 n} & =a \sum_{k=1}^{n} \frac{(2 n-2) !}{(2 k-2) !(n-k) !(n-k+1) !}(b-a)^{n-k}(v-u)^{2 k-2}, \\
r_{2 n+1} & =a \sum_{k=1}^{n} \frac{(2 n-1) !}{(2 k-1) !(n-k) !(n-k+1) !}(b-a)^{n-k}(v-u)^{2 k-1} .
\end{aligned}
$$

Proof. Putting $s_{m}:=s_{m}(\mu(a, b, u, v))$ we have from the Bożejko-Accardi formula:

$$
s_{m}=\sum_{\sigma \in \mathrm{NC}_{1,2}(m)} a^{\text {out }_{2}(\sigma)} u^{\text {out }_{1}(\sigma)} b^{\mathrm{inn}_{2}(\sigma)} v^{\mathrm{inn}_{1}(\sigma)},
$$

where out $2(\sigma)$, out $_{1}(\sigma), \operatorname{inn}_{2}(\sigma), \operatorname{inn}_{1}(\sigma)$ denotes the number of outer or inner blocks $V \in \sigma$, with $|V|=2$ or $|V|=1$, respectively. For fixed $\sigma \in \mathrm{NC}_{1,2}(m)$ the related 
summand can be written as

$$
a^{\text {out }_{2}(\sigma)} u^{\text {out }_{1}(\sigma)}((b-a)+a)^{\operatorname{inn}_{2}(\sigma)}((v-u)+u)^{\operatorname{inn}_{1}(\sigma)} .
$$

After expanding we get a sum of products of factors of the form $a, u,(b-a),(v-u)$, which can be described in terms of signed noncrossing partitions. By a signing of $\sigma \in \mathrm{NC}_{1,2}(m)$ we will mean a function $\epsilon: \sigma \rightarrow\{0,1\}$ such that $\epsilon(V)=0$ whenever $V \in \sigma$ is an outer block. We will denote by $\operatorname{Sign}(\sigma)$ the family of all signings of $\sigma$. Now we define the weight of a signed block:

$$
w(V, \epsilon):=\left\{\begin{array}{lr}
u \quad \text { if }|V|=1 \text { and } \epsilon(V)=0, \\
v-u \text { if }|V|=1 \text { and } \epsilon(V)=1, \\
a \quad \text { if }|V|=2 \text { and } \epsilon(V)=0, \\
b-a \text { if }|V|=2 \text { and } \epsilon(V)=1,
\end{array}\right.
$$

and the weight of a signed partition:

$$
w(\sigma, \epsilon):=\prod_{V \in \sigma} w(V, \epsilon) .
$$

Then the expansion of the product (18) can be written as

$$
\sum_{\epsilon \in \operatorname{Sign}(\sigma)} w(\sigma, \epsilon)
$$

Now, for a fixed pair $(\sigma, \epsilon)$, with $\sigma \in \mathrm{NC}_{1,2}(m), \epsilon \in \operatorname{Sign}(\sigma)$ we define a partition $\Pi(\sigma, \epsilon)$ by gluing a block $V$ with $V^{\prime}$ whenever $\epsilon(V)=1$. More precisely, define a relation $\mathcal{R}$ on $\sigma: U \mathcal{R} V$ iff $V=U^{\prime}$ and $\epsilon(U)=1$. Let $\sim$ be the smallest equivalence relation on $\sigma$ containing $\mathcal{R}$. Then we define a partition $\Pi(\sigma, \epsilon)$ of $\{1,2, \ldots, m\}$ whose blocks are of the form $\bigcup \mathcal{C}$, with $\mathcal{C} \in \sigma / \sim$. This means that $k$ and $l$ are in the same block of $\Pi(\sigma, \epsilon)$ if and only if there are blocks $U, V \in \sigma$, with $k \in U, l \in V$, and numbers $r, s \geq 0$ such that

$$
\begin{aligned}
& \epsilon(U)=\epsilon\left(U^{\prime}\right)=\cdots=\epsilon\left(U^{(r-1)}\right)=1, \\
& \epsilon(V)=\epsilon\left(V^{\prime}\right)=\cdots=\epsilon\left(V^{(s-1)}\right)=1
\end{aligned}
$$

and $U^{(r)}=V^{(s)}$. It is easy to see that $\Pi(\sigma, \epsilon)$ is noncrossing.

On the other hand, for fixed $\pi \in \mathrm{NC}(m)$, we have $\Pi(\sigma, \epsilon)=\pi$ if and only if $\sigma$ is finer than $\pi$ (i.e. for every $V \in \sigma$ there is $U \in \pi$ such that $V \subseteq U$ ) and for every $U \in \pi$, if $U=\left\{k_{1}, k_{2}, \ldots, k_{r}\right\}, k_{1}<k_{2}<\cdots<k_{r}$, then we have $\left\{k_{1}, k_{r}\right\} \in \sigma$, with sign 0 , and all the blocks of $\sigma$ which are contained in $\left\{k_{2}, \ldots, k_{r-1}\right\}$ have sign 1 . In particular, every one-element block $U \in \pi$ must be a block of $\sigma$ with sign 0 . Therefore the sum of weights $w(\sigma, \epsilon)$ with $\Pi(\sigma, \epsilon)=\pi$ is equal to

$$
\sum_{\substack{\sigma \in \mathrm{NC}_{1,2}(m), \epsilon \in \operatorname{Sign}(\sigma) \\ \Pi(\sigma, \epsilon)=\pi}} w(\sigma, \epsilon)=u^{N_{1}(\pi)} \prod_{\substack{U \in \pi \\|U|>1}} \sum_{\sigma_{U} \in \mathrm{NC}_{1,2}(|U|-2)} a(v-u)^{N_{1}\left(\sigma_{U}\right)}(b-a)^{N_{2}\left(\sigma_{U}\right)},
$$

where $N_{1}(\sigma), N_{2}(\sigma)$ denotes the number of one- and two-element blocks in $\sigma$. Hence

$$
\begin{aligned}
s_{m} & =\sum_{\sigma \in \mathrm{NC}_{1,2}(\mathrm{~m})} a^{\text {out }_{2}(\sigma)} u^{\text {out }_{1}(\sigma)} b^{\mathrm{inn}_{2}(\sigma)} v^{\mathrm{inn}_{1}(\sigma)} \\
& =\sum_{\sigma \in \mathrm{NC}_{1,2}(\mathrm{~m})} a^{\text {out }_{2}(\sigma)} u^{\text {out }_{1}(\sigma)}((b-a)+a)^{\operatorname{inn}_{2}(\sigma)}((v-u)+u)^{\operatorname{inn}_{1}(\sigma)}
\end{aligned}
$$




$$
\begin{aligned}
& =\sum_{\pi \in \mathrm{NC}(\mathrm{m})} u^{N_{1}(\pi)} \prod_{\substack{U \in \pi \\
|U|>1}} \sum_{\tau \in \mathrm{NC}_{1,2}(|U|-2)} a(v-u)^{N_{1}(\tau)}(b-a)^{N_{2}(\tau)} \\
& =\sum_{\pi \in \mathrm{NC}(m)} \prod_{U \in \pi} c_{|U|}
\end{aligned}
$$

where $c_{1}=u$ and for $m \geq 2$

$$
c_{m}=a \cdot \sum_{\tau \in \mathrm{NC}_{1,2}(m-2)}(v-u)^{N_{1}(\tau)}(b-a)^{N_{2}(\tau)} .
$$

Since the numbers $c_{m}$ satisfy the same recurrence relation (5) as $r_{m}$, we have $r_{m}=s_{m}$ for every $m \geq 1$. It is well known that the number of those $\pi \in \mathrm{NC}(2 j)$ for which $|V|=2$ for every $V \in \pi$ is equal to the Catalan number $\frac{1}{j+1}\left(\begin{array}{c}2 j \\ j\end{array}\right)$. Hence the number of partitions $\sigma \in \mathrm{NC}_{1,2}(m)$ with $i$ blocks of order one and $j$ blocks of order two, $i+2 j=m$, is equal to $\left(\begin{array}{c}m \\ i\end{array}\right) \frac{1}{j+1}\left(\begin{array}{c}2 j \\ j\end{array}\right)$, which leads to the coefficients in (10) and (11).

Observe that the subclass $\{\mu(a, 0, u, v): a>0, u, v \in \mathbb{R}\}$ coincides with the family of two-point probability measures on $\mathbb{R}$, namely:

$$
\mu(a, 0, u, v)=p_{-} \delta_{x_{-}}+p_{+} \delta_{x_{+}}
$$

where

$$
\begin{aligned}
& p_{ \pm}=\frac{\sqrt{(u-v)^{2}+4 a} \pm(u-v)}{2 \sqrt{(u-v)^{2}+4 a}}, \\
& x_{ \pm}=\frac{u+v \pm \sqrt{(u-v)^{2}+4 a}}{2} .
\end{aligned}
$$

and, on the other hand,

$$
\begin{aligned}
& a=p_{+} p_{-}\left(x_{+}-x_{-}\right)^{2}, \\
& u=p_{+} x_{+}+p_{-} x_{-}, \\
& v=p_{+} x_{-}+p_{-} x_{+} .
\end{aligned}
$$

Corollary. For $a, b>0, u, v \in \mathbb{R}$ and $t \geq 0$ the free power $\mu(a, b, u, v)^{\boxplus t}$ exists if and only if $b-a+t a \geq 0$ and then

$$
\mu(a, b, u, v)^{\boxplus t}=\mu(t a, b-a+t a, t u, v-u+t u) .
$$

In particular, $\mu(a, b, u, v)$ is infinitely divisible if and only if $a \leq b$.

If $0 \leq b<a$ then $\mu(a, b, u, v)$ is a free power of a two point measure:

$$
\mu(a, b, u, v)=\mu\left(a-b, 0, \frac{a-b}{a} u, v-u+\frac{a-b}{a} u\right)^{\boxplus \frac{a}{a-b}} .
$$

Proof. The first statement holds because one can see from the theorem that for every $m \geq 1$

$$
r_{m}(\mu(t a, b-a+t a, t u, v-u+t u))=t \cdot r_{m}(\mu(a, b, u, v)) .
$$

The rest is an obvious consequence. 


\section{References}

[AB] L. Accardi and M. Bożejko, Interacting Fock spaces and Gaussianization of probability measures, Infin. Dimens. Anal. Quantum Probab. Relat. Top. 1 (1998), 663-670.

[BB] M. Bożejko and W. Bryc, On a class of free Lévy laws related to a regression problem, J. Funct. Anal. 236 (2006), 59-77.

[Ch] T. S. Chihara, An Introduction to Orthogonal Polynomials, Gordon and Breach, 1978.

[CT] J. M. Cohen and A. Trenholme, Orthogonal polynomials with a constant recursion formula and an application to harmonic analysis, J. Funct. Anal. 59 (1984), 175-184.

[SY] N. Saitoh and H. Yoshida, The infinite divisibility and orthogonal polynomials with a constant recursion formula in free probability theory, Probab. Math. Statist. 21 (2001), 159-170.

[S1] R. Speicher, A new example of "independence" and "white noise", Probab. Th. Rel. Fields 84 (1990), 141-159.

[S2] R. Speicher, Multiplicative functions on the lattice of non-crossing partitions and free convolution, Math. Ann. 298 (1994), 611-628.

[Vi] G. Viennot, Une théorie combinatoire des polynômes orthogonaux généraux, Lecture Notes, UQAM, 1983. 